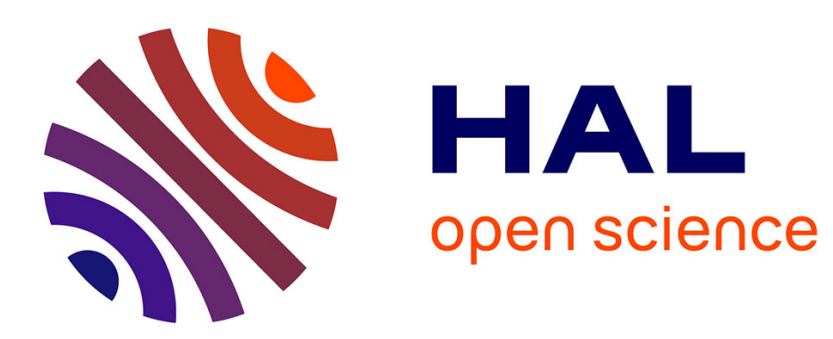

\title{
What Is the Use of Diagrams in Theoretical Modeling? Anouk Barberousse
}

\section{To cite this version:}

Anouk Barberousse. What Is the Use of Diagrams in Theoretical Modeling?. Science in Context, 2013, 26 (2), pp.345 -362. 10.1017/S0269889713000082 . halshs-01058668

\section{HAL Id: halshs-01058668 \\ https://shs.hal.science/halshs-01058668}

Submitted on 2 Sep 2021

HAL is a multi-disciplinary open access archive for the deposit and dissemination of scientific research documents, whether they are published or not. The documents may come from teaching and research institutions in France or abroad, or from public or private research centers.
L'archive ouverte pluridisciplinaire HAL, est destinée au dépôt et à la diffusion de documents scientifiques de niveau recherche, publiés ou non, émanant des établissements d'enseignement et de recherche français ou étrangers, des laboratoires publics ou privés. 
What is the use of diagrams in theoretical modelling?

Anouk Barberousse

\section{Introduction}

Diagrams and all types of doodles are widely used in theoretical physics. They help physicists create new models and understand their implications. However, the exact way physicists work with graphic representations is still poorly understood. The main reason why the analysis of non verbal scientific representations is still at its beginnings is that we lack sophisticated tools for understanding the semantic properties of these representations, while we already possess a rich toolbox for the analysis of formal languages, and, to a lesser extent, natural language. Moreover, it is not sure that the semantic analysis of diagrams, understood as the study of the conditions at which they can be seen as conveying meaning about a situation, can be unified. Even though research is very active about the use of diagrams in logic (see for instance Chandrasekaran et al., 1995, Hammer, 1995, Shin, 1994) and is developing about the use of diagrams in mathematics (Mancosu et al., 2005), it is difficult to apply its results when it comes to empirical sciences because more aspects are involved, including visual appearance and resemblance, whose role in the epistemology of pictures in general is highly controversial (Lopes, 1996, Hopkins, 1998).

The purpose of the present paper is to undertake an investigation upon diagrams' and doodles' content and the impact they may have on scientific practice. The main claim of the paper is that diagrams efficiently contribute to the cognitive process coming off in the understanding of a theoretical model in physics. In order to establish this claim, I provide an 
analysis of the role of diagrams in the practice of theoretical modelling. This analysis relies on a fictionalist account of models based on Walton's approach.

The diagrams and doodles drawn by theoretical physicists, either during the research phase, when teaching, or in papers and textbooks, are usually highly ambiguous in the sense that they cannot be understood unless heavily relying on background information. For instance, when a physicist displays a diagram during a talk, he always points to some parts of it and gives its audience the relevant instruction to understand it clearly. In papers and textbooks as well, the legend and the cross-references to the figures in the text constitute obligatory supplementation to the figures. Physicists' diagrams do not stand on their own in the sense that they cannot be understood independently of the legend and text, and have thus to be accompanied by pieces of natural language (sometimes connecting them to mathematical formulae or equations). For sure, this feature of non verbal representations does not disjoin them from linguistic representations, since hardly any single sentence in a physics paper does stand on its own either. Usually, it cannot be understood unless the reader possesses a lot of information about the topic of the paper. Diagrams and linguistic representations are thus comparable with respect to their lack of semantic and epistemic autonomy when they are part of a paper in physics. Admittedly, sentences form linguistic units and are easier to analyse as such than diagrams from the semantic point of view; however, from the readers' point of view, both sentences and diagrams are impossible to grasp without relevant background information. So, neither diagrams nor sentences stand on their own, as both lack autonomy in the sense that they depend on context in order to be interpreted and understood.

Nevertheless, it has long been taken for granted by historians and philosophers of science that non verbal representations are redundant vis à vis the text and that they cannot be understood 
without it. For instance, Baigrie regrets the "privilege given to theory in the image of science inherited from logical positivism" (1996, xvii): "scientific knowledge has traditionally been taken to be encapsulated in theories which, in turn, are interpreted by the logical empiricists as axiomatic systems. The primary mode of scientific representation for the logical empiricists is linguistic." He adds that "despite the publication of a number of important historical studies on the role of pictorial devices in particular sciences during the last quarter century (see Rudwick, 1976 and Ferguson, 1977), the textual deposits of scientists and scientific illustration are still generally regarded by science studies scholars as fundamentally different modes of expression. [...] Coupled with the deep-seated conviction that human thinking takes place in words, the supposition persists that the pictures in science are psychological devices that serve as heuristic aides when reasoning breaks down. [...] What distinguishes pictorial devices as resources for doing science, and the special problems that are raised by the mere presence of visual elements in scientific treatises, tend to be eclipsed by philosophical worries about the nature of representation (ibid., xviii). Giere develops this point further: "In the framework of logical empiricism, $[\ldots]$, there can be no fundamental role in science for non-linguistic entities like pictures or diagrams. Such things might, of course, play some part in how scientists actually learn or think about particular theories, but unless their content is reduced to linguistic form, they cannot appear in a philosophical analysis of the content or legitimacy of any scientific claims to knowledge" $(1996,270)$. He insists that "Kuhn himself did not highlight the role of visual or other non-propositional modes of representation in science" (ibid.). As a consequence of this past situation, diagrams and pictures have not been studied as interesting objects of historical or philosophical enquiry until recently (among the recent papers taking the role of scientific pictures seriously, let us mention Kayser, 2005; Lynch, 1988; Lopes, 2009; Perini, 2004, 2005a, b, c, d). 
It remains that physicists make a massive use of diagrams and doodles. The present paper investigates how they impact scientific practice. It assumes that the elaboration of theoretical physics is not only a matter of mathematics and other formalized languages and that much has to be gained from the systematic study of various representational formats, as Humphreys (2004) convincingly argues. Humphreys does not focus on the pictorial format in his book, but his claim that "syntax matters" may be applied to pictures as well as to equations: in the same way as finite difference equations differ from differential equations, a naturalistic line drawing of a teal differs from a photograph of a teal of the same species. These differences are highly relevant to the role scientists assign to equations, resp. to pictures.

In Section 2, I present examples in which diagrams do participate in the building up of the model and, consequently, in the transmission of its meaning. Section 3 is devoted to an analysis of the role of diagrams in the practice of theoretical modelling. In order to elaborate such an analysis, I rely on a fictionalist account of models. In Section 4, I show the advantages of the fictionalist approach.

\section{Examples}

The diagrams I am going to analyse in this section are commonly used in polymer science since the 1970s. They are not pictures of actual polymer chains, the relevant features of which are not observable, but of idealized chains. They thus display theoretical models of the behavior of these chains in various circumstances. 


\subsection{The self-similar grid}

The diagram reproduced in figure 1 represents, as indicated in the original caption, an (idealized) adsorbed polymer layer. A polymer is a macromolecule, that is, a molecule with a high molecular weight. It is made out of a sequence of several macromolecules (typically several hundreds) called "monomers", which are the chemical units of the polymer chain. The polymer chain, when put into a liquid (in a solution) can be imagined as a very long noodle.

Polymers have numerous industrial applications. In order to improve them, it is important to have a detailed knowledge of the chains' behavior in various situations. Among the situations which manufacturers want to better control, the interfaces, that is, the contact surfaces between a solution of polymer and a solid, or air, have been studied extensively. Theoretical accounts of the behavior of polymers at an interface are rather recent, but already substantial. Polymer chains are said to be "adsorbed" when some of them, those which are close to the interface with a solid surface, get attracted to it, and hang on to it. As the detailed behavior of polymer chains at an interface cannot be seen through a microscope, because it occurs in three dimensions, how and why the chains are adsorbed is a matter of theoretical investigation. Moreover, the validity of the involved hypotheses cannot be assessed in any simple way by currently available observations.

The diagram presented in figure 1 is part of a theoretical model of adsorption in which the concentration of adsorbed polymer chains increases as they get closer to the surface. This model has been proposed by P.-G. de Gennes (1932-2007), a theoretical physicists who has mainly worked in the field of condensed-matter physics. He won the Nobel prize in 1991 for his pioneering works on liquid crystals and polymer physics. In the paper from which the diagram is taken, he puts forward (among other achievements) a new theoretical model of adsorption. This 
model was entirely different from the ones that had been proposed beforehand, which tried to describe the adsorption phenomenon from complex calculations based on the analysis of the individual motions of the polymer chains. By contrast, De Gennes first figures out the global geometrical aspect of the solution in the vicinity of the interface. In this new approach, the goal in to discover the ratio to which the concentration of adsorbed polymer chains increases as they get closer to the wall. The ratio is both given a a graphical representation in the diagram and symbolic representation in the text, in the form of a power law of ratio $a / z, a$ being the size of a monomer, and $z$ the distance to the wall. The diagram thus presents the geometrical structure of the adsorption schema.

Let me now analyse the way in which the diagram displays the logic of the adsorption phenomenon. First, it is important to notice that the diagram represents the variation of the polymer chains' density relative to the distance from the interface. The geometrical structure of the diagram is a "self-similar grid", which means that the ratios among the sizes of the diagram's cells is the same throughout the whole grid, except for their size: on the diagram, the left half of each cell is cut into two equal cells, and this construction procedure goes on like that (this is what "self-similarity" means) until the interface is reached. Anyone familiar with the notion of self-similarity will recognize it just by taking a quick look at the diagram. Moreover, the notion of self-similarity is contained in the power law which is at the heart of the mathematics of the model. Thus, the diagram appears to be "just" a graphical expression of the power law equation. But is it really?

The foregoing figure in the paper (here, figure 2) graphically explains the idealization governing the making up of the diagram presented in figure 1. Whereas polymer chains build up supple entanglements when placed into a solution (as shown on the left of the figure), the 
structure they form can be represented in a more mathematically tractable way by replacing their noodle-like aspect by a grid composed of straight lines (as shown on the right of the figure). Replacing the curved shape of the polymer chains by straight lines allows for a simpler geometrical treatment.

It has to be further noted that figure 2 is self-sustaining as a vehicle of meaning (for someone who already possesses the background knowledge about polymer physics that is needed to understand the paper). Its content is not expressed otherwise anywhere in the paper. As it is critical to understand it in order to fully understand the diagram of the self-similar grid, it can be said that this diagram contains a part of the content of the paper which is not repeated in the text: someone who understands figure 2 will also understand that figure 1 explains why polymer chains can be seen as building up a self-similar grid when they get adsorbed at an interface. According to De Gennes' hypothesis in the paper, they indeed get entangled in such ways as to build up a concentration gradient idealized by a self-similar grid.

To sum up, this example shows that a diagram (figure 1), coming with its generation procedure (figure 2), may have a richer content than the equation it comes along with. The diagram in figure 1 is not just a graphical display of a mathematical formula, since it also contains a physical hypothesis about how polymer chains get entangled near an interface: the physical hypothesis is that the adsorption phenomenon follows a power law, represented by the structure of the self-similar grid.

\subsection{Grafted surfaces}


Figure 3 shows two more models of the behavior of polymer chains at an interface. The surface in contact with the polymer solution is "grafted", namely, it has been prepared in such a way that the polymer chains get chemically attached to it (and not only adsorbed). Two grafted regimes are hypothesized through these images: the "brush regime" (Figure 3 (a)) and the "mushroom regime" (Figure 3 (b)). These diagrams shows the behaviors of polymer chains in two limiting situations in good solvents: the "brushes" appear when the grafting density is high, whereas the "mushrooms" are hypothesized when the grafting density is low. So, the solution at low densities is modelled in the following way: each chain is isolated, occupying roughly a halfsphere with a radius comparable to the radius of gyration. At high densities, polymers stretch away from the interface to avoid overlapping, forming a "brush". Even though the words "mushroom" and "brush" strongly suggest the way the polymer chains are arranged close to the wall, the diagrams add important information. Let me quote the paper: "In good solvent conditions, the chains repel each other and the grafting reaction terminates when the chains are disposed like adjacent "mushrooms" [reference to the figure]. In some favorable cases, on can push the density of grafted points to higher values; the result is the "brush" of [the figure], with a relatively thick region of constant concentration" (De Gennes, 1987, 192). The diagram conveys information about the meaning of the non-overlapping condition: without it, one may have imagine that the chains could stretch further from the wall in the low density regime, for instance.

What is thus remarkable in these examples is the interplay between the drawings and the metaphors ("brush", "mushroom") coming from everyday language. This interplay provides the models' users with a mnemonic device helping them to keep track of the difference between the various hypothesized regimes. 
3. What theoretical models represent

The examples presented in Section 2 show that diagrams can be on the same footing as mathematical and linguistic representations when theoretical models are introduced and discussed. Why is it so? Two complementary answers may be given to this question. (i) It is only a matter of cognitive ease; only using mathematical and linguistic expressions would be two awkward, and cognitively difficult to handle. (ii) Diagrams do make a positive contribution to the presentation and understanding of theoretical models; they allow for a richer and more precise presentation $^{1}$. I shall first elaborate on the implications on answer $(i)$ and then begin an argument in favor of answer (ii) that will go on in Section 4. The starting point of this argument is a discussion of the nature of theoretical models.

\subsection{About the cognitive tractability of diagrams}

The use of diagrams in physics may be attributed both a virtue and a vice: first, they help physicists to communicate quicker than by means of complicated equations, this is the virtue; second, the price of easiness of cognitive access is vagueness, conceived as an evil. In his severe criticisms against models and modeling in chapter 4 of his book, Pierre Duhem (1906)

\footnotetext{
${ }^{1}$ Throughout the paper, I am using "presentation" or "mode of presentation" as meaning the way a concept, or any content, is given to us. For instance, the thought that there is a cat on the mat is presented here by written language, but it may also occur in your mind when you look at a painting of a cat on a mat, or a photography, or else when someone tells you that there is a cat on the mat.
} 
denounces this very point and claims (in his own terms) that vagueness is too high a price for cognitive ease. Duhem does not focus on diagrams; he rather contrasts (British) models and (French and German) abstract theories. However, Maxwell's and Thomson's papers are full of diagrams, so that Duhem's criticism may legitimately be conceived as applying to diagrams as well as models. Let me analyze the balance between cognitive ease and vagueness further in this section.

Why are diagrams so efficient in conveying information about what they represent? The main reason is that our visual system is particularly apt to process two-dimensional (as well as three-dimensional) information (see for instance Kennedy, 1974; Cavanagh, 1995). The ubiquity of drawing in all human cultures is probably due to this physiological and cognitive characteristics. Our capacity to process visual information is much used in everyday life. There is no reason why it should not be used in scientific activity, except for ideological reasons (as an example of such a denial of images, one can mention the group of French mathematicians Bourbaki, who forcefully forbids the use of pictures in mathematics).

From a cognitive-science perspective, easiness of understanding is an advantage of diagrams; however, their use in mathematics and physics is commonly associated with the cognitive limitations of human beings, as if easiness was deemed to come along with potential misunderstandings. In this common view, diagrams are just temporary expedients that are only used in contexts where mathematical rigor is either not necessary because everyone in the audience could introduce it if needed, or not yet available. In the latter case, diagrams are provisional and indicate a road toward a more complete formulation, whereas in the former, they are used as reminders of the more rigorous formulation. The traditional perspective on diagrams thus does not take them seriously, as genuine vehicles of information. It assumes that physicists 
make doodles when they cannot find the right words, equations, or even concepts, or that these doodles are only cognitive aids, without playing any role of their own.

The traditional view on diagrams is based on another widely shared assumption according to which content is most adequately conveyed by language, more precisely by formal language. In this view, formal language is conceived as the ideal mean of representation and communication. For sure, the defenders of this view admit that scientists do not only, or even mostly, communicate with formal language, but they interpret this fact as a sign that, as human beings, scientists are cognitively limited. Another consequence of the traditional view is that diagrams are seen as merely helping readers understand propositions that are already expressed in a text, or that could at least be expressed linguistically. However, we would like to know how diagrams help people understand the parts of physics in which they appear (Laura Perini has proposed an interesting hypothesis in the case of some models in biology: Perini, 2005b, c).

\subsection{The nature of theoretical models}

In section 3.1 , I have examine answer $(i)$ to the question why diagrams are as capable as mathematical and linguistic representations to introduce theoretical models: because their use is cognitively easier than the use of equations. I now turn to the second answer, according to which diagrams make a positive contribution to the presentation and understanding of theoretical models because they allow for a richer and more precise presentation. In this section, my aim is to analyse how diagrams are involved in the modeling activity. Relying on the examples presented in section 2, I shall show that diagrams are even indispensable in the modeling 
practice. In order to fulfill this aim, I first turn to a proposal about the nature of theoretical models.

Theoretical models are used in virtually every field of science, but no analysis of their nature has been proposed yet, upon which there would be a general agreement. On the one hand, they are usually seen as too heterogeneous to be analysed on common grounds; on the other hand, the historical and philosophical literature on models is despairingly copious (see for instance Black, 1962, de Chadarevian and Hopwood, 2004, Frigg, 2008, Giere, 2004, GodfreySmith, 2006, Hughes, 1997, 1999, Morgan and Morrison, 1999). In this section, I only rely on a small part of it in order to present a view of models that provides a conceptual framework in which the role of diagrams in modeling is better understood than in other views.

Following Nancy Cartwright's claim that "models are works of fiction" (Cartwright, 1983, p. 153), I claim (Barberousse and Ludwig, 2009) that a theoretical model is a representation of a fictional situation (other fictionalist positions with respect to models are presented in Woods, 2010 and Suarez, 2009). The main argument in favor of this thesis is that models often represent situations of which we know that they are impossible, according to our best theories of the phenomena in question. The prima facie paradox arising from the claim that scientists play with fictions vanishes when it is added that these fictions are related with the investigated phenomena through intricate relationships of idealization and approximation. The main point of theoretical modelling, and its main difficulty, is to evaluate the validity of the hypotheses justifying the use of such and such idealization or approximation in given cases. This is a notoriously difficult task (cf., e.g., Redhead, 1980; Laymon, 1991). Even the plausibility of these hypotheses is usually hard to assess. In most cases, we do not know how to describe, in a precise and unproblematic fashion, the relationships between the fictional situation represented 
by a model and the experimental situation the meaning and structure of which the model is supposed to make clear, for instance by explaining some aspects of it. This is the reason why diagrams may also have a use in suggesting new experiments: as their meaning is not entirely fixed, they may prompt new ways of investigating the phenomena they aim at representing, ways that were not used before. ${ }^{2}$

The above characterization of models has to be complemented with an account of their epistemic value and fruitfulness. In order to achieve this goal, let us first describe the cognitive relationship we entertain with fictions. From the point of view of the producers and users of fictions, a fiction is a representation whose function is to make its interpreter imagine a certain content. A novelist, for instance, implicitly asks her readers to develop a certain type of attitude towards the propositions expressed in the narration. The required type of attitude, which we, as readers, usually adopt without effort, is the attitude of make-believe or imaginative implication (Currie, 1990, p. 18). To put it in a nutshell, the intention of the novelist is that the reader makes believe that the story is true.

In reading novels or watching movies, we adopt the attitude of make-believe for the sake of entertainment. In working on theoretical models, by contrast, we look for explanations or other epistemic goods. Needless to say, the imaginative game is severely constrained by this quest. Here is an attempt at describing it: the scientific-imaginative game consists in bracketing the concepts that lead to contradictions between the model and commonly accepted theories within the relevant field. These contradictions are the reason why models are often qualified as literally false representations (see for instance Redhead, 1980; Godfrey-Smith, 2008). They are indeed literally false about the experimental situations they contribute to describe and explain;

\footnotetext{
${ }^{2}$ I am grateful to Christoph Hoffmann for suggesting me this point.
} 
however, to leave off there is unsatisfactory. It is the epistemic fruitfulness of models that requires explanation, not their bare falsehood. In order to account for the fruitfulness of models in spite of their being literally false, let us take a simple example. In so-called "billiard ball" models of gases, the term "molecule" takes a different meaning from the meaning it has in our best physicochemical theories: in the model, "molecule" refers to perfectly elastic spheres, whereas in our best physical theories about molecules, they are far from being elastic. Thus, what the model implies about molecules contradicts what the best available theories of molecules say they are. However, more has to be said about the meaning of the word "molecule" in the billiardball model than mentioning this contradiction. What is more important than the bland contradiction is that the meaning of the concept of molecule is left partially indeterminate within the model. When exploring the implications of the model, we only assume that "molecules" are perfectly elastic spheres, without knowing, e.g., how atoms are combined within such spheres. Generally speaking, models are (sometimes) the way to theoretical innovation precisely because the meanings of some concepts within them are left indeterminate. This important feature sets the rules of the game of scientific make-belief in modeling.

The above-presented conception of models relies on the commonly accepted assumption that the meaning of a concept depends on its context of use (cf. for instance Preyer and Peter, 2005). In the case of fiction, the context of use includes the imaginative project of the interpreter. Now, the interpreter's project, that is, its aim in entering the fiction, participates in determining the meaning of the very concepts that are used in the description of the imagined situation. For instance, when reading a novel from Jane Austin, the reader's knowledge and expectations about nineteenth century English society influence her understanding of the plausibility of the ongoing engagements. Contemporary readers' plausibility appraisal were probably slightly different from 
ours. The construction of theoretical models proceeds in the same way: they are made up of concepts the meaning of which is not the usual one. It is not completely fixed, which gives way to (controlled) theoretical innovation.

An important consequence of this conception of theoretical models is that when we grasp a model, as when we look at or read an artistic fiction, part of our concepts are reorganized. This reorganization is sometimes deep and upsetting, for instance when a student discovers the specificities of quantum physics ${ }^{3}$. However, it may be more benign. My point here is that as soon as one is willing to try and understand the content of a model, she is also willing, consequently, to accept a (sometimes provisional) change in her epistemic framework. Some relationships between concepts may be transformed, as well as the very meaning of these concepts. For instance, physicists working with the so-called "liquid-drop model of the nucleus", which is a semi-empirical model (both relying on currently accepted fundamental principles, and on relationships that have been empirically established), have to (provisionally) change their view on electromagnetic forces and nuclear forces within the nucleus relative to what the standard model, which is the best available theory of elementary particles, implies.

The conceptual reorganization required by the use of models is often guided by words or other symbols acquiring new meanings as they appear in the model. For instance, the words "brush" and "mushroom" acquire a new meaning when they are used within De Gennes' model of grafted polymers. The new contents symbols acquire within models have two important peculiarities: $(i)$ they are given by idiosyncratic concepts, which are built up within the model itself and (ii) they are not always expressed by words or other symbolic notations, but may also

\footnotetext{
${ }^{3}$ I warmly thank Christoph Hoffmann for suggesting me that the concept reorganization may have various impacts according to the epistemic context the subject is engaged in.
} 
be presented by means of diagrams. In the next section, I give an account of the way diagrams may contribute to the content of theoretical models.

\section{Diagrams as parts of models}

In section 3, I have presented a fictionalist framework in which models are analysed as fictions. Within this framework, it is the cognitive abilities of scientists to handle fictional situation that account for the fruitfulness of models in spite of their being literally false about the investigated situations. The aim of the present section is to show that this approach makes the role of diagrams in modeling clearer. I first present an analysis of how diagrams may contribute to modeling that is based on Walton's work. In section 4.2, I provide illustrations of this analysis in two contexts where scientific diagrams are used: teaching and research.

\subsection{Diagrams as "props"}

In a nutshell, the main claim I am going to argue for in this section is that diagrams, like equations and verbal descriptions, are tools promoting the capacity of scientists to imagining the situation represented by the model. When seeing and understanding a diagram, a scientist is guided toward the idealized situation it points to, in the same way as a written sentences steers the reader's mind toward the situation it describes, be it real or fictional. The difference between a diagram and a sentence is that usually, a diagram is worth several sentences. 
Let us now investigate the ways in which a diagram facilitates cognitive access to a model. The first point to notice is that as a model represents a fictional, that is, unreal situation, there are aspects in it that are radically different from the situations we are acquainted with, either through perception or through theoretical knowledge. Already known situations are of limited guidance when one is trying to grasp a new, theoretical model. More precisely, as we saw in section 3 , the fictional situation represented by a model usually conflicts with the implications of the best available theories in the field. For this reason, the fictional situation is especially difficult to figure out -- even by the designers of the model. In this context, which is highly specific to scientific activity because a model, even though accessible only by imagining a fictional situation, is nevertheless strictly constrained by the requirement of empirical adequacy, diagrams may play a distinguished role: they may be more efficient than sentences, either in formal or natural languages, to guide the formation of an imagined situation in the reader's mind, that would be compatible with the available information on the model.

In the context of the current theories of imagination, the role I just described for diagrams has been termed "prop" by Walton (1990). I thus claim that diagrams presenting theoretical models (on a par with equations and verbal descriptions) may be analyzed as "props", or "generators of fictional truths". Here is how Walton characterizes props:

Props are generators of fictional truths, things which, by virtue of their nature of existence, make propositions fictional. [...] A doll makes it fictional in a child's game that there is a blonde baby girl. Representational works of art are props also. (pp. 37-38).

Diagrams, as well as mathematical and linguistic expressions, may usefully be considered as props guiding the physicists' minds toward the model's theoretical content, and helping them to figure out what this content may be. Before coming back to the examples given in section 2 in 
order to give more flesh to this analysis, let me note that this interpretation is corroborated by Walton's description of the role of props:

The role of props in generating fictional truths is enormously important. They give fictional worlds and their contents a kind of objectivity, an independence from cognizers and their experiences [...] (p. 42).

Can the above presented diagrams be analysed as props? Let us first focus on Figure 2. By first giving a picture of entangled polymer chains that is close to what a realistic picture could be, Figure 2 is aimed at convincing the reader that the diagram on the right, which is the building block of the diagram in Figure 1, is nothing but a further idealization. It does not add or retrieve anything important. The function of Figure 2 is thus to induce in the reader's mind the conception that the idealization on the right, and thus the basic components of Figure 1, are scientifically acceptable. Let me add that this conception would be very awkward to express by words (even the ongoing comment is much clumsier that the figure!): a wordy paraphrase would claim that it is plausible to idealize the basic element of polymer chain entanglement as a rectangle. But it may immediately be collected that the paraphrase looks arbitrary, whereas the diagram is a plausible idealization.

Let us now turn to Figure 3. For sure, the verbal metaphor "brush" and "mushroom" can already be considered as props: they guide the reader's mind toward grafted regimes that are neither random nor entirely ordered. However, the diagrams make the reader understand that not every imaginable "brush" or "mushroom" are meant. They give details about the mushroom's size and about the layout of the brush's hairs, so to speak. Whereas the equations describing the two limiting regimes only bear on quantifiable properties of the polymer chains, the diagrams give an idea of their spatial layout. What is important here is that the spatial layout can only be 
imagined, not exactly represented; relying on this specificity, the diagrams strongly add to the model's plausibility. May it be only a sort of rhetorical effect, if the extension of the term "rhetoric" to diagrams is allowed? It is difficult to answer this question. What is testified, however, is that both the metaphors and the diagrams are commonly used by now in papers investigating the properties of grafted chains of polymers. At least, the diagrams are props in the sense that they give the model "a kind of objectivity", detaching it from its inventor, and allowing inter-subjective reference.

I have just sketched an analysis of diagrams as props, considering them in the same way as sentences in natural or formal languages in scientific papers. The question may arise of the nature of the difference between diagrams and sentences with respect to this function. The above examples can guide us toward possible answers. Take figure 2 . The schema on the left manifests itself, so to speak, as a first idealization, for real polymer chains can hardy be found in such a configuration. To put it differently, it generate the fictional truth that polymer chains implement this configuration. The right part of the figure exhibits how this configuration can be further idealized. This part, when complemented with Figure 1, generates in turn the fictional truth that the mathematical treatment of the phenomenon in terms of a power law is plausible. It seems hard to find out sentences that play this two-step role so efficiently. This may be because we are dealing with geometrical idealizations. The explanation of the epistemic power of diagrams may thus rest on the human mind's specific ability to interact with (simple) geometrical figures.

Figure 3, however, drives us toward a different explanation. One of its main characteristics is that it suggests that the detailed constitution of the "mushrooms", that is, the precise way polymer chains are gathered on the surface, does not matter. What counts is that there are gathered into clearly separated "mushrooms". Besides their capacity to express 
geometrical properties, diagrams thus have the power to suggest indeterminacy, contrary to a widely shared view about pictures, which holds that pictures are always more determinate than sentences. These remarks suggest in turn that the epistemic power of diagrams, as opposed to sentences, will probably be revealed when the semantic and pragmatic properties of pictures in general will be disclosed, e.g. in the ongoing work of Lopes (1996), Hopkins (1998), Kulvicki (2006), Willats $(1997,2003)$, etc.

4.2 Various uses of diagrams: teaching vs. research

Analyzing diagrams as props helping physicists imagine the fictional situations represented by models allows for an easy understanding of their pedagogical role. In this respect, the following two questions are to receive different answers:

(1) How do diagrams express theoretical content?

(2) How do diagrams express new theoretical content?

Let us look at an example of a pedagogical diagram. In figure 4, a common, pedagogical presentation of Bohr's model of the atom is displayed. (In his original paper 1913, Bohr only wrote sentences and equations, but his model of the atom is usually presented with the help of diagrams in introductory lessons on quantum mechanics). In this diagram, the ellipses representing the supposed trajectories of the electrons around the nucleus are immediately associated, by any physics student, with the trajectories of planets around the Sun. The association of the ellipses with the usual graphical representation of planetary motion emphasizes the fictional character of the situation represented by the model, since according to the best 
available electromagnetic theories available at Bohr's time, the electrons must collapse on to the nucleus instead of describing elliptical orbits. Thus, the background knowledge of electrons having a negative electric charge and of protons having a positive one conflicts with the perception of the ellipses. This is what makes the situation represented by Bohr's model fictional: it is not compatible with the accepted picture of the microscopic world at the time. The reason why Bohr postulated in his model that electrons did not collapse, in contradiction with the accepted electromagnetic theories, is that this allowed him to explain recently discovered empirical phenomena concerning the inner structure of atoms.

In this example, we can understand how diagrams are used within pedagogical contexts, that is, how to answer question (1) as opposed to question (2). In a classroom or in a textbook, the meaning of every line in the diagram has a clear explicit and implicit meaning for both the teacher and the students who understand what the lesson is about. This is the reason why the diagram helps students figure out the content of the model by relying of their previous experiences of looking at other diagrams in the physics class.

Diagrams of new theoretical models, on the other hand, as the ones presented in section 2 , are used for prospective, not only pedagogical, purpose. They represent theoretical content that is not completely understood yet, that is, the meaning of the new concepts building up the model is open. The interpretation of these diagrams surely relies on previously experienced images in the same domain, but in a way that leaves their meaning partially open.

\section{Conclusion}


Diagrams representing theoretical models are pervasive in all branches of theoretical physics. Contrary to what could be inferred from the usual reconstructions of philosophers of science, equations do not reign supreme over papers presenting theoretical models. Diagrams sometimes convey an important part of the theoretical content expressed in these papers. When models are interpreted as fictions, diagrams can be conceived as "props" generating theoretical content in their interpreters' minds. This conception helps understand the differential roles of diagrams in pedagogical contexts and in the presentation of new theoretical content.

\section{Acknowledgment}

Many people helped me refine my ideas in previous versions of this paper: Dominic Lopes, Alfred Nordmann, Laura Perini, and Catherine Allamel-Raffin. Marion Vorms, Alexandre Guay, Cyrille Imbert, Vincent Israel-Jost, and Christohp Hoffmann provided me with helpful comments on the penultimate version. I wish to warmly thank all of them, as well as two anonymous referees who forced me to clarify many aspects.

\section{Bibliography}

Baigrie, Brian, ed. 1996. Picturing Knowledge: Historical and Philosophical Problems Concerning the Use of Art in Science, University of Toronto Press.

Barberousse and Ludwig. 2009. "Models as fictions". In Suarez, ed., 2009, 56-73. 
Black, Max. 1962. Models and Metaphors. Ithaca, New York: Cornell University Press.

Bohr, Niels. 1913. "On the constitution of atoms and molecules". Philosophical Magazine, 26: $1-25,476-502,857-875$.

Cartwright, Nancy. 1983. How the Laws of Physics Lie. Oxford: Oxford University Press.

Cavanagh, Patrick. 1995. "Vision is getting easier everyday", Perception (24): 1227-1223.

Chandrasekaran, Bharath, Glasgow, J., and Narayanan, N. Hari, (eds.). 1995, Diagrammatic Reasoning: Cognitive and Computational Perspectives. Cambridge, MA: AAAI Press/The MIT Press.

de Chadarevian, Soraya and Nick Hopwood. 2004. Models: The third dimension of science. Stanford, CA: Stanford University Press.

Currie, Gregory. 1990. The Nature of Fiction, Cambridge: Cambridge University Press.

Duhem, Pierre. 1906. La Théorie physique, son objet, sa structure. Paris: Gauthier-Villars. English translation: The Aim and Structure of Phyiscal Theory, Princeton University Press, 1991. 
Ferguson, Eugene S. 1977. "The Minds Eye: Non-Verbal Thought in Technology". Science, 197 (4306): 827-836.

Frigg, Roman. 2009. "Models and fictions". Synthese, 172(2), 2010, 251-268.

De Gennes, Pierre-Gilles. 1981. "Polymer solutions near an interface. 1. Adsorption and depletion layers". Macromolecules: 14.

De Gennes, Pierre-Gilles. 1987. "Polymers at an interface. A simplified view". Advances in colloid and interface science: 27.

Giere, Ronald N. 1996. "Visual Models and Scientific Judgment", in Baigrie, ed., 269-302.

Giere, Ronald N. 2004. "How models are used to represent reality". Philosophy of Science, 71: $742-752$.

Godfrey-Smith, Peter. 2006. "The strategy of model-based science". Biology and Philosophy, 21: $725-740$.

Godfrey-Smith, Peter. 2008. "Abstractions, idealizations, and evolutionary biology". In Barberousse, A., Morange, M. and Pradeu, T. (eds) Mapping the Future of Biology: Evolving 
Concepts and Theories. (Boston Studies in the Philosophy of Science), Springer, 2009, pp. 47-56.

Hammer, Eric, 1995. "Reasoning with Sentences and Diagrams", Notre Dame Journal of Formal Logic, 35(1): 73-87.

Hopkins, Robert. 1998. Picture, Image, and Experience. Cambridge: Cambridge University Press.

Hughes, Richard I. G. 1997. "Models and representation". Philosophy of Science, 64: S325S336.

Hughes, Richard I. G. 1999. "The Ising Model, Computer Simulation, and Universal Physics". In Models as Mediators. Perspectives on Natural and Social Science, edited by Mary S. and Margaret Morrison, 97-145. Cambridge UK: Cambridge University Press.

Humphreys, Paul. 2004. Extending ourselves. Computational Science, Empiricism and Scientific Method. Oxord: Oxford University Press.

Kayser, David. 2005. Drawing Theories Apart. The Dispersion of Feynman Diagrams in Postwar Physics. Chicago: The University of Chicago Press. 
Kennedy, John M. 1974. A Psychology of Picture Perception, San Francisco, CA: Jossey-Bass Publishers.

Kulvick, John. 2006. On Pictures: Their Structure and Content. Oxford, Clarendon Press.

Laymon, Richard. 1991. "Computer simulations, idealizations and approximations". PSA 1990, vol. 2: 519-534.

Lopes, Dominic M. 1996. Understanding Pictures. Oxford: Clarendon Press.

Lopes, Dominic M. 2009. "Drawing in a Social Science: Lithic Illustration", Perspectives on Science, 17: 5-25.

Lynch, Michael. 1988. "The externalized retina: Selection and mathematization in the visual documentation of objects in the life sciences", Human Studies 11:201-234.

Morgan, Mary S. and Margaret Morrison (eds.). 1999. Models as Mediators. Perspectives on Natural and Social Science. Cambridge UK: Cambridge University Press.

Mancosu, Paolo, K. Jørgensen and S. Pedersen, eds., 2005. Visualization, Explanation and Reasoning Styles in Mathematics, Berlin: Springer. 
Perini, Laura. 2004. "Convention, Resemblance and Isomorphism: Understanding Scientific Visual Representations," in Multidisciplinary Approaches to Visual Representations and Interpretations, ed. Grant Malcom, Elsevier.

Perini, Laura. 2005a. "Explanation in Two Dimensions: Diagrams and Biological Models," Biology \& Philosophy, 20(2-3): 257-269.

Perini, Laura. 2005b. "The Truth in Pictures," Philosophy of Science 72(1): 262-285.

Perini, Laura. 2005c. "Visual Representations and Confirmation," Philosophy of Science 72(5): 913-926.

Perini, Laura. 2005d. "Scientific Reasoning, Mental Models, and Depiction". In Art and Cognition Workhosp «Pictures in Cognition and Science », http://www.interdisciplines.org/ $\underline{\operatorname{artcognition}}$

Preyer, Gerhard, and Peter, Georg (eds). 2005. Contextualism in Philosophy. Knowledge, Meaning, and Truth, Oxford University Press.

Redhead, Michael. 1980. "Models in Physics". British Journal for the Philosophy of Science, 31: $145-163$. 
Rudwick, Martin J. S. 1976. The Meaning of Fossils: Essays in the History of Paleontology, Elsevier, 2nd ed.

Shin, Sun-Joo, 1994. The Logical Status of Diagrams. Cambridge: Cambridge University Press.

Suarez, Mauricio. 2009. Fictions in Science: Philosophical Essays on Modeling and Idealisation, London: Routledge.

Thomson-Jones, M. (2005). Idealization and abstraction: A framework. In M. Thomson-Jones, \& N. Cartwright (Eds.), Idealization XII: Correcting the model, idealization and abstraction in the sciences, 173-217.

Walton, Kendall. 1990. Mimesis as Make-Believe. On the Fondations of the Representational Arts. Harvad: Harvard University Press.

Willats, John. 1997. Art and Representation. Princeton: Princeton University Press.

Willats, John. 2003. Optical laws or symbolic rules? The dual nature of pictorial systems. In H. Hecht, R. Schwartz, and M. Atherton (eds.) Looking into Pictures. Cambridge, MA: MIT Press: 125-144.

Woods, John. 2010. Fictions and Models. Philosophia Verlag GmbH. 
Pedagogical presentation of Bohr's model of the atom: http://theory.uwinnipeg.ca/physics/bohr/ node3.html 


\section{Figures}

Figure 1. Diagram of the concentration gradient of polymer chains in solution at an interface. (De Gennes P.-G. 1987 « Polymers at an interface. A simplified view », Advances in colloid and interface science, 27)

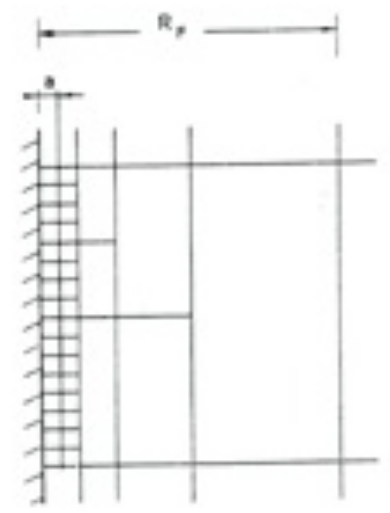

Pig. 7. An adsorbed polymer layer represented as a "self-similar grid". At any distance, $z$, from the wall, the local mesh size is equal to $\mathrm{z}$.

Figure 2. Idealization procedure explaining the construction of figure 1 (Ibid.).

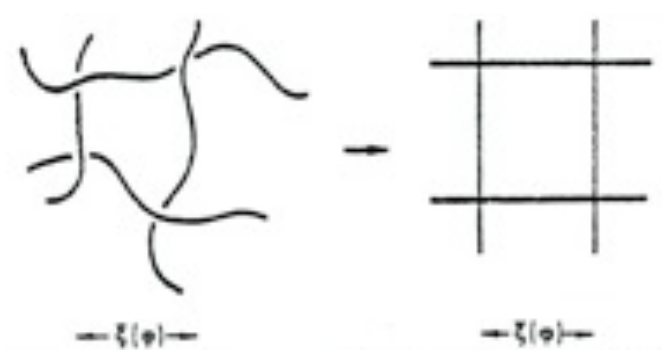

Fig. 6. A polymer solution (volume fraction 4 ) idealized as a "grid" with the same mesh size, $t(\bullet)$.

$\xi=\mathbf{a} \bullet^{-3 / 4}$, 
Figure 3. Grafted surfaces.
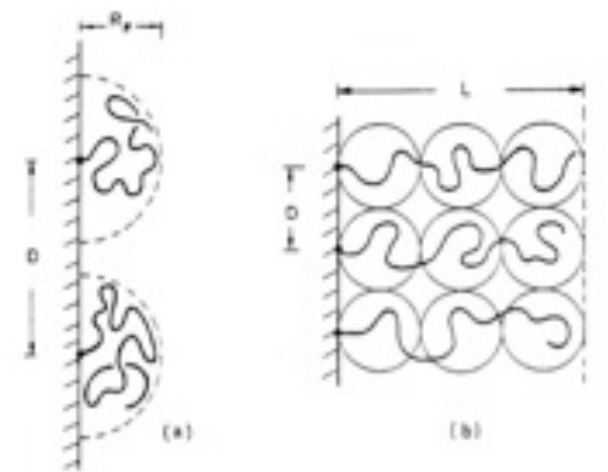

(b)

Fig. 4. Two types of grafted surfaces: a) low grafting density -- the distance between heads D is larger than the coil size Rp (this is the "mushroom" regime); b) high grafting density $D<R_{F}$ ) (the "brush").

3. Use of block copolymers (ref. 1). If a chain is made of one insoluble part A (the "anchor") and one soluble part B (the "buoy"), it will often

Figure 4. Planetary model of the atom, pedagogical presentation Bohr's model (source: http://theory.uwinnipeg.ca/physics/bohr/node3.html) 


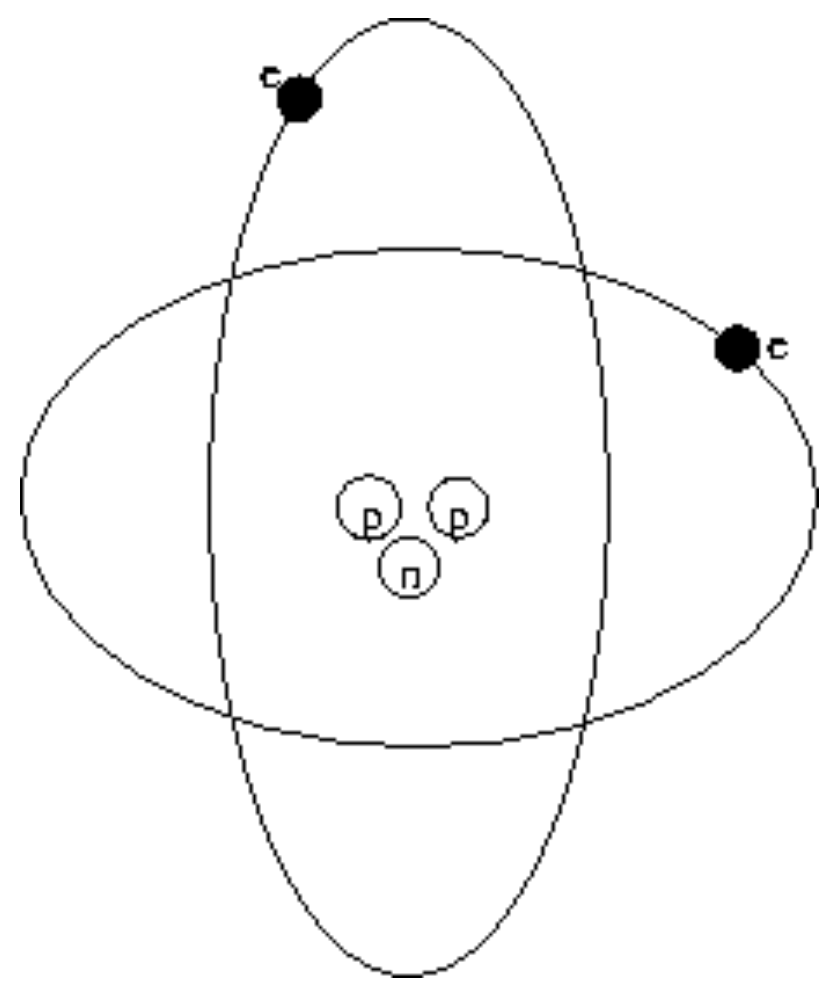

